\title{
Kippbilder. Wie sich Aspekte heraus-bilden
}

»düte knapp zwöi zyle y vrschidensti richtige /

u när sy dy verschidenste dy richtige ${ }^{1}$

Wie mit Ambiguität umgehen? Nicht nur Bilder, sondern auch einzelne Worte und kurze Zeilen können in der Wahrnehmung der Rezipierenden kippen oder oszillieren. In manchen Fällen mag es sich dabei um einen erwünschten Effekt lyrischer Produktion handeln. Alltäglicher ist die knapp zweizeilige Textnachricht, die die Empfängerin, den Empfänger gewollt oder ungewollt quält. Nämlich, weil sie entweder jenes oder dieses bedeuten kann, aber nicht beides auf einmal - obwohl beide Deutungen »richtig« sind.

In solcher Not wünschte man sich vielleicht, es gäbe ein zuverlässiges Verfahren, Ambiguität zu reduzieren und Eindeutigkeit herzustellen. Könnte hier nicht die Hermeneutik als \Lehre vom Verstehen abhelfen? Eine solche Hoffnung wird sich zerschlagen. Wer verstehen möchte, wie verstanden wird, merkt bald: Je breiter und tiefer die eigene Kenntnis hermeneutischer Theorie und Praxis, desto vielfältiger die Bedeutungsnuancen und -aspekte, die sich zeigen. Hermeneutik bietet vieles, aber keine Erlösung von Ambiguität. Ingolf $U$. Dalferth formuliert es so:

"Die Pointe des hermeneutischen Prozesses ist [...] nicht die Desambiguierung von Sinn, sondern gerade umgekehrt die Steigerung von Sinnambiguität. Es geht nicht um die Aufdeckung des reigentlichen< Sinns, sondern um die Öffnung des Sinn- und Verstehenshorizonts, ohne dass unterstellt oder erwartet werden könnte, Mehrfachverstehen und Mehrfachsinn könnten irgendwann in einem Verständnis und einem Sinn zum Ende kommen. $\mathbb{2}^{2}$

Mehr noch: Wer die 'Kunst des Verstehens` erlernen möchte, muss Ambiguität geradezu begrüssen. Denn, so Dalferth weiter: "Das

\footnotetext{
1 [»Deute knapp zwei Zeilen in verschiedenste Richtungen / Und danach sind die Verschiedensten die Richtigen "] Das Zitat stammt aus dem Song Hollywood Was Talking About Love Before Anyone Else Did der Band Jeans for Jesus (dies., Jeans for Jesus, 2014 bei Tune Kid Records). Der phonetische Kippeffekt dieser beiden Zeilen funktioniert nur in Berner Mundart, in der der ganze Song geschrieben ist - hier klingen der Plural von >Richtung und der des substantivierten Adjektivs srichtig` genau gleich.

2 Ingolf U. Dalferth, Die Kunst des Verstehens. Grundzüge einer Hermeneutik der Kommunikation durch Texte, Tübingen 2018, 501.
} 
hermeneutische Ziel ist nicht, dass am Ende alle alles gleich verstehen, sondern das [sic!] jeder das, was er versteht, auch noch anders versteht, nämlich so, wie es andere verstehen, und dass er es dadurch besser versteht, als er es allein von sich aus hätte verstehen können. « ${ }^{3}$

Thomas Bauer zieht in seinem Essay Die Vereindeutigung der Welt diese Linie weiter aus. Er warnt vor einer zunehmenden Einebnung spannungsvoller Ambiguitäten nicht nur in Kunst, Literatur und Musik, sondern auch persönlichen Beziehungen sowie im gesellschaftlichen und politischen Diskurs. Um dem entgegenzuwirken sei "Ambiguitätstraining «" angezeigt. Übrigens eine Forderung, die das lyrische Ich aus dem oben zitierten Songtext möglicherweise mitunterschreiben würde. Trotz allen hermeneutischen Tücken des Versuchs, per Whatsapp (vielleicht?) eine Beziehung anzubahnen, befindet es einige Zeilen weiter:

"i bi kes barbie mit sprachchip /

dy maschine het kei geist, ke seelä /

dy fähler woni mache sy dy eigetä « ${ }^{5}$

Entgegen diesen Plädoyers war (semantische) Ambiguität begriffsgeschichtlich längst nicht nur positiv besetzt. Gerade antiken Denkern, die nach differenzierten Kategorien, rhetorischer Klarheit und logischer Stringenz strebten, war Uneindeutigkeit zuwider. ${ }^{6}$ Es muss hier offen bleiben, wann und aus welchen Gründen sich diese Haltung zu ändern begann und eine hermeneutische Lust an der Ambiguität sich mehr und mehr verbreitete. ${ }^{7}$ Auf der Hand liegt aber, dass Ambiguität eine Art Grundbegriff all jener philosophischen Strömungen geworden ist, die gegenüber einem ungebroche-

\footnotetext{
3 Ebd.

4 Thomas Bauer, Die Vereindeutigung der Welt. Über den Verlust an Mehrdeutigkeit und Vielfalt, Stuttgart ${ }^{132} 2018,95$.

5 ["Ich bin keine Barbie mit Sprachchip / Diese Maschine hat keinen Geist, keine Seele / Die Fehler, die ich mache, sind die eigenen «] Jeans for Jesus, Hollywood Was Talking About Love Before Anyone Else Did.

${ }^{6}$ Vgl. Wolfgang Ullrich, Grundrisse einer philosophischen Begriffsgeschichte von Ambiguität, in: Archiv für Begriffsgeschichte 32 (1989), 121-169, hier 122-132.

7 Vgl. aber etwa die Hinweise auf die diesbezügliche Rolle Nietzsches ebd., 153155. Wichtig auch zu bedenken, dass >Ambiguität in diesem Zusammenhang eine neue Beziehung erhielt.Vgl. ebd., 164: "Es ist offensichtlich, daß im 20. Jhdt. Ambiguität als philosophischer, wahrnehmungspsychologischer oder ästhetischer Begriff eine bedeutende Rolle spielt, wobei jeweils der streng sprachliche Bezug, der bis in die beginnende Neuzeit herrschend war, verlorengeht, um eher sekundär und abgeleitet zur Geltung zu kommen. Diese Umbeziehung des Begriffs bedeutet zugleich, daß nicht mehr die Vermeidung von Zweideutigkeiten als Ziel proklamiert wird.»
} 
nen Erkennen von Gegenständen die Perspektivität, das `Sehen-als stark machen. Und in diesem Zusammenhang sind Kippbilder und -figuren ein Phänomen, an dem sich der hermeneutische Umgang mit Ambiguität kristallisiert. ${ }^{8}$

Kippbilder lassen sich in verschiedensten Anwendungsfeldern entdecken. Manche Beispiele reichen historisch weit zurück; ${ }^{9}$ heute berühmte Kippbilder haben ihren Ursprung dagegen in Zeitschriften des 19. und frühen 20. Jahrhunderts, wo sie als Scherzbilder veröffentlicht wurden. Aus einer solchen Zeitschrift übernahm der Psychologe Joseph Jastrow den Hasen-Enten-Kopf und machte ihn für die Erforschung der menschlichen Wahrnehmung nützlich. ${ }^{10}$ Sein 1899 publizierter Artikel über The Mind's Eye enthielt noch zahlreiche weitere Kippfiguren. Jastrow bezeichnete sie als "ambiguous diagrams" oder "ambiguous figures «. 1921 erschien Edgar Rubins Studie Visuell wahrgenommene Figuren, auf die weitere berühmte, oft variierte Kippbilder zurückgehen - allen voran die RubinscheVase. ${ }^{12}$ Seither hat die Wahrnehmungs- und Kognitionspsychologie eine schier unüberblickbare Menge an Kippbildern unterschiedlicher Gattungen für ihre Zwecke entdeckt (oder gleich selbst entwickelt). Einige davon sind in diesem Heft abgebildet.

Ein wichtiger Katalysator der stärker philosophisch orientierten Rezeption von Kippbildern waren Wittgensteins Philosophische Untersuchungen. Seine u.a. an den Hasen-Enten-Kopf anschliessenden Überlegungen ${ }^{13}$ haben diesen zu einer Art Emblem hermeneutischer

\footnotetext{
8 Vgl. ebd., 156.

9 Vgl. etwa Marco Piccolino/Nicholas J. Wade, Flagging Early Examples of Ambiguity I, in: Perception 35 (2006), 861-864 sowie dies., Flagging Early Examples of Ambiguity II, in: Perception 35 (2006), 1003-1006.

${ }^{10}$ Joseph Jastrow, The Mind's Eye, in: Popular Science Monthly 54 (1899), 299-312, hier 312. Vgl. ebd., 310 Jastrows Bemerkungen zu diesem Kippbild: "Somewhat different, but still belonging to the group of ambiguous figures, is the ingenious conceit of the duck-rabbit [...]. When it is a rabbit, the face looks to the right and a pair of ears are conspicuous behind; when it is a duck, the face looks to the left and the ears have been changed into the bill. Most observers find it difficult to hold either interpretation steadily, the fluctuations being frequent, and coming as a surprise.«Vgl. ferner Peter Brugger, One Hundred Years of an Ambiguous Figure: Happy Birthday, Duck/Rabbit!, in: Perceptual and Motor Skills 89.3 (1999), 973-977.

${ }^{11}$ Jastrow, The Mind's Eye, 307.310.

12 Edgar Rubin,Visuell wahrgenommene Figuren. Studien in psychologischer Analyse, Kopenhagen u.a. 1921.

${ }^{13}$ Vgl. Ludwig Wittgenstein, Philosophische Untersuchungen. Philosophical Investigations. English and German, trans. Gertrude E.M. Anscombe, Oxford/Malden 1999 (1953), 194.
} 
Forschung gemacht. ${ }^{14}$ Seither dienen Kippbilder gerade auch in der Philosophie zur Reflexion über Erkenntnis und Wahrnehmung. Auch für religionsphilosophische oder theologische Gedankengänge bieten sie zahlreiche Anschlussmöglichkeiten. Die Denkpotenziale, die Wittgensteins Kippbild-Reflexionen bieten, sind bei Weitem noch nicht ausgeschöpft. Sie helfen zu verstehen, wie wir verstehen und wie Andere, seien es Texte, Bilder oder Menschen, sich uns zu verstehen geben - oder eben nicht. Es überrascht daher nicht, dass sich mehrere Beiträge dieses Hefts mit Wittgenstein befassen und von ihm ausgehend eigene kippbildhermeneutische Einsichten entwickeln.

Was aber macht eigentlich ein Kippbild aus? Eine umfassende Definition soll hier nicht versucht werden. ${ }^{15}$ Ein vorläufiger Vorschlag, mit dem sich weiterarbeiten lässt, könnte aber etwa so aussehen: Kippbildern gemein ist die Eigenschaft, dass sie den Augen der Betrachterinnen und Betrachter zunächst als ein Bild von etwas erscheinen. Erst auf den zweiten Blick entpuppt sich dasselbe Bild auch als etwas anderes. Was zunächst als ein Ganzes erschien (z.B. der Kopf einer Ente), tritt nun auseinander in zwei Aspekte (einerseits Hasen-, andererseits Entenkopf), von denen keiner dem anderen gegenüber in irgendeiner Weise prioritär ist. $\mathrm{Ab}$ diesem Moment ist das Bild erst als Kippbild benennbar. Im Gegensatz zu anderen Bildern zeichnet sich ein Kippbild gerade dadurch aus, dass es nicht in eine reigentlicher Abbildung und einen suneigentlichen<, zweiten Aspekt unterteilt werden kann, wie das etwa der Fall wäre bei dem Bild einer Wolke, in der man mit etwas Phantasie auch ein Gesicht erblicken kann. Das /Wesen <es Kippbildes erscheint also weder im einen noch im anderen Aspekt, sondern im Moment des Kippens.

Charakteristisch für ein Kippbild ist ferner der hermeneutische Weg, den man bei seiner `Lektüre zurücklegt und hinter den man nicht mehr zurück kann. Bei anderen Bildern addiert man neu entdeckte Details und Ansichten gleichsam zum Ganzen des Bildes hinzu. Das ist bei einem Kippbild anders: Ist erst einmal ein zweiter Aspekt entdeckt, das Bild als Kippbild erkannt, so lässt sich beim Sehen des einen Aspekts nicht mehr vergessen, dass er eben nur ein

\footnotetext{
${ }^{14}$ Vgl. etwa das Coverbild bei Andreas Mauz/Christiane Tietz (Hg.),Verstehen und Interpretieren. Zum Basisvokabular von Hermeneutik und Interpretationstheorie, Paderborn 2020.

${ }^{15}$ Für eine ausführlichere bildtheoretische Einordnung vgl. Rainer Schönhammer/ Jakob Steinbrenner, Kippbild, in: Glossar der Bildphilosophie, hg. v. Jörg R.J. Schirra/ Mark A. Halawa/Dimitri Liebsch (2012-2021) - URL: http://www.gib.uni-tuebingen.de/netzwerk/glossar/index.php?title=Kippbild\&oldid=21393 (Zugriff: 10. August 2021).
} 
Aspekt und nicht das Ganze ist - ja, dass es das Ganze so nicht mehr gibt. Denn im Gegensatz zu svorher, als der zuerst gesehene Aspekt nicht als Aspekt, sondern als das ganze Bild erschien, kann das Bild jetzt nur noch hinsichtlich seines Kippens zwischen beiden Aspekten als Ganzes verstanden werden. Aber die beiden Aspekte lassen sich nie gleichzeitig sehen. ${ }^{16}$

Einige weitere hermeneutische Grössen wiederum beeinflussen das `Lesen eines Kippbilds ganz ähnlich, wie sie auf die Rezeption anderer Bilder oder Texte einwirken. Mitunter eignet sich das Kippbild besonders, um deren Wirkung offenzulegen. Das gilt etwa in Bezug auf die Intentionalität eines Werks: Schon Jastrows erste Experimente mit dem Hasen-Enten-Kopf zeigten, dass sich recht genau bestimmen lässt, bei welchen Variationen des Motivs sich ein eigentlicher Kippbild-Effekt einstellt, also mit hoher Wahrscheinlichkeit sowohl der Hasen- als auch der Enten-Kopf gesehen wird. ${ }^{17}$ Das heisst umgekehrt: Kippbilder lassen sich intentional als solche herstellen. ${ }^{18}$ Die Intentionalität spielt nicht nur auf Seite der Bildproduzierenden, sondern auch der -rezipierenden: Sind die zwei (oder mehr) Aspekte des Kippbildes erst einmal entdeckt, so lässt sich über das Moment des Kippens oft willkürlich verfügen.

Umgekehrt gibt es auch das Phänomen des Nicht-Verstehens oder des Unverständnisses, nämlich wenn einem ein Kippbild als solches verschlossen bleibt: Es kann sein, dass nur ein Enten- und

\footnotetext{
${ }^{16}$ Vgl. dazu ausführlicher das Kapitel Spaltende Bilder. Das Kippbild als hermeneutisches Modell für das Verständnis von Dezentrierungen in: Luca Di Blasi, Dezentrierungen. Beiträge zur Religion der Philosophie im 20. Jahrhundert, Wien/Berlin 2018, 39-64. Im Zusammenhang mit dem Kippbild zu erwähnen ist auch die Bildtechnik der Anamorphose. Dank Techniken der perspektivischen Verzerrung geben anamorphotische Bilder einzelne in ihnen abgebildete Gegenstände oder Ansichten den Betrachtenden nur zu erkennen, wenn diese aus einer bestimmten Perspektive oder mithilfe optischer Geräte auf das Bild blicken. Im Unterschied zur hier skizzierten Definition eines Kippbildes verlangt ein anamorphotisches Bild also eine räumliche Veränderung des Standpunkts und/oder eine technische Erweiterung der Wahrnehmung der Rezipierenden - und reflektiert diese Faktoren der Bildrezeption somit in seinem Bildaufbau. Dies wiederum gibt Anlass zu weiterführenden kunsttheoretischen und philosophischen Reflexionen.Vgl. Yvonne Schweizer, Anamorphose, in: Glossar der Bildphilosophie, hg. v.Jörg R.J. Schirra/Mark A. Halawa/Dimitri Liebsch (2012-2021) - URL: http://www.gib.uni-tuebingen.de/netzwerk/glossar/index.ph p?title $=$ Anamorphose\&oldid $=21307$ (Zugriff: 10 . August 2021).

${ }_{17}$ Vgl. Brugger, One Hundred Years, 975.

${ }^{18}$ Schön illustriert das etwa die Sammlung von Hase-Ente-Kopf-Kippbildern des Psychologen John F. Kihlstrohm: https://www.ocf.berkeley.edu/ jfkihlstrom/JastrowDuck.htm (Zugriff: 10. August 2021).
} 
kein Hasenkopf gesehen wird - oder umgekehrt. Wittgenstein nannte das " Aspektblindheit «" ${ }^{19}$. Wer aspektblind ist, sieht nicht `nur einen Aspekt des Kippbildesı, sondern nur ein Bild (z.B. eines Entenkopfs), das gerade nicht als Kippbild erkannt wird. Es stellt sich dann die Frage, ob dieser Blindheit abgeholfen werden muss und kann.

Katalysator und Vehikel des Denkens: Das Kippbild gibt somit Gelegenheit, die Zwischenräume und Wendepunkte, die Unschärfen und Ambiguitäten auszuloten, die sich einstellen, wenn wir wahrnehmen und verstehen (möchten). Es erfüllt dabei zwei Funktionen, die in Wechselwirkung stehen. Zum einen dient es als Anstoss und Gegenstand des Nachdenkens über solche Kipp-Phänomene. Zum anderen ist es ein Vehikel, das es erlaubt, solche Phänomene in allerlei philosophischen, theologischen und literarischen Werken, aber ebenso sozialen, kulturellen sowie politischen Ordnungen und identity markers $\mathrm{zu}$ greifen und zu reflektieren.

Die Ausgabe $27 \mid 1+2 \cdot 2021$ der Hermeneutischen Blätter erkundet diese Funktionen von Kippbildern. ${ }^{20}$ Die Beiträge kommen dabei, wie für diese Zeitschrift üblich, aus unterschiedlichen Disziplinen. Entsprechend vielfältig sind die Zugänge, die sie wählen. Dennoch ergeben sich bei der Lektüre immer wieder spontane Querverbindungen zwischen ihnen. Damit bewahrheitet sich einmal mehr, was eingangs mit Dalferth festgehalten wurde: Dass umfassenderes, besseres Verstehen nicht aus der "Aufdeckung des >eigentlichen< Sinns" erwächst, sondern aus der "Öffnung des Sinn- und Verstehenshorizonts«. So trägt dieses Heft hoffentlich dazu bei, das Phänomen des Kippbilds und was es über unser Verstehen zu verstehen gibt, in noch mehr Aspekten darzustellen.

Möglich gemacht haben diese Ausgabe die Autorinnen und Autoren der Beiträge. Ihnen allen sei herzlich gedankt. Im Hintergrund tätig für redaktionelle Aufgaben und Layout war Susanne Schenker. Auch ihr gilt ein grosser Dank. Die vorliegende Ausgabe der Hermeneutischen Blätter, soviel ist nach den vorstehenden Überlegungen

\footnotetext{
${ }_{19}$ Wittgenstein, Philosophische Untersuchungen, 213.

${ }^{20}$ Mehrere Sammelbände haben sich bereits der Thematik gewidmet, allerdings mit anderen Schwerpunkten als dieses Heft: Vgl. Christoph F. E. Holzhey (Hg.), Multistable Figures. On the Critical Potential of Ir/Reversible Aspect-Seeing, Wien/Berlin 2014. (Zum Download unter: https://www.ici-berlin.org/oa/ci-08/index.html [Zugriff: 10. August 2021]); Kippfiguren/Figures réversibles, hg. v. Hans-Georg von Arburg/Marie Theres Stauffer: figurationen. gender - literatur - kultur 13.2 (2012); Kay Junge u.a. (Hg.), Kippfiguren. Ambivalenz in Bewegung, Weilerswist 2013.
} 
deutlich, will für ihre Leserinnen und Leser die Ambiguität von Kippbildern gerade nicht reduzieren. Umso mehr wünsche ich ihnen eine eindeutig anregende Lektüre.

Dominik von Allmen-Mäder 